\title{
A Case Report of Human Demodicosis in a Patient Referred to a Dermatology Clinic in Tabriz, Iran
}

\author{
Hesamoddin Mohebbi*, Shayan Boozarjomehri Amniyeh, Parisa Mahdavi, Ali Heydari Azar Heris \\ Department of Parasitology, Tabriz Branch, Islamic Azad University, Tabriz, Iran
}

\begin{abstract}
Background: The genus Demodex belongs to the order Prostigmata and the family Demodecidae that has several species of uncommon mites, some of which cause severe scabies in animals. There are two species of this mite that cause disease in humans, including Demodex folliculorum, which is known as hair follicle mite, and Demodex brevis. This disease is more common in women than in men.

Case Presentation: The patient is a 36-year-old woman living in one of the villages of Tabriz city who referred to a dermatologist following severe itching and hyperkeratosis (abundant dandruff) of the cheeks. Then, she was introduced to the laboratory for preparing a slide from a sample taken from the patient's cheeks. A large number of Demodex mites were observed in the microscopic test of the sample.

Conclusion: In patients referred to skin clinics with scaling and itching, especially in the head and face, the complication may be due to Demodex infection. Therefore, it is suggested that demodicosis be considered in differential diagnosis in such patients. Keywords: Skin, Hyperkeratosis, Human demodicosis, Tabriz city
\end{abstract}

Received: December 1, 2019, Accepted: December 15, 2019, ePublished: January 1, 2020

\section{Introduction}

The genus Demodex belongs to the order Prostigmata and the family Demodex that has several species of uncommon mites, some of which cause scabies in animals. Two species of the genus Demodex are pathogenic in humans and are more common in women than in men. They include Demodex folliculorum, known as the hair follicle mite, and Demodex brevis, known as the sebaceous glands (lipid glands) mite (1).

In differential diagnosis, the size of this mite is very small $(0.3-0.4 \mathrm{~mm})$. It has a striped abdomen. Generally, it is not like a mite, but rather it is relatively similar to a worm. It has a very small chest and four pairs of fat legs with five segments (2).

This mite is not mistaken for any of the other mites that infect humans. Demodex is found in hair follicles, sebaceous glands, eyelids, nose and around it, cheeks, ear secretions (ear wax), and small pimples and feed on skin secretions, especially sebum (3).

The disease is rare in people under 10 years of age and is more common in people over 40 years of age. Demodex can cause dermatitis, acne-like pimples, infectious yellowwound, or eyelid inflammation, and it usually does not appear to have side effects (4).

To diagnose the disease, the scales of suspicious areas can be gently scraped with a scalpel and collected on a slide, using one or two drops of a clear solution such as potassium hydroxide (10\%-20\%), glycerin or lactophenol for microscopic examination.

Female mites lay eggs at the base of the hair, and the larvae emerge from them, which turn into nymphs and eventually into adults after metamorphose. All growth stages (13-15 days) take place in the hair follicles or sebaceous glands. In severe infections that lead to dermatitis, drugs containing polysulfide may be used (5).

These drugs should not be rubbed into the eyelid as they may cause irritation. Other treatments include $0.5 \%$ selenium sulfide ointment, $10 \%$ sulfur ointment, and daily washing with soap and water can reduce the contamination $(6,7)$.

\section{Case Presentation}

The patient is a 36-year-old woman living in one of the villages of Tabriz city who was introduced to the laboratory of Sina hospital in Tabriz, Iran, by a dermatologist in June 2017 for testing. The lesions observed in the cheek area were relatively severe dermatitis with extensive crusting and itching. According to the patient, her symptoms started three weeks before referral. The symptoms included the appearance of a few small pimples on the cheeks. Then, they expanded and she referred to the doctor due to redness and itching of the skin. After sampling and preparation of slides from the species, a large number of Demodex mites (4-5 in each microscopic field) were observed (Figure 1). 


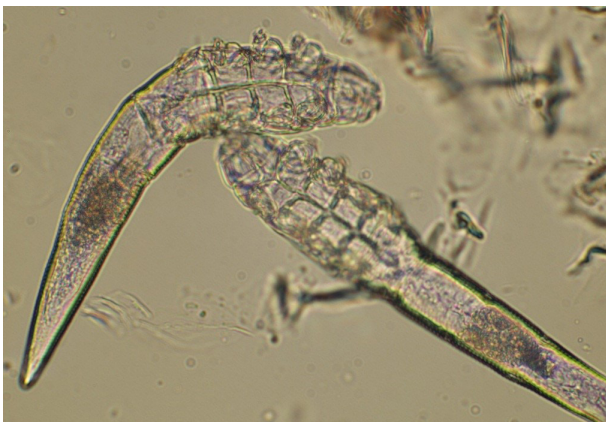

Figure 1. Demodex, the Causative Agent of Human Demodicosis

\section{Discussion}

Demodex is one of the uncommon mites in human infections and has different species that can cause severe complications in animals, especially dogs. This mite can be one of the important factors in the development of rosacea and dermatitis.

The presence of Demodex folliculorum in diseases such as acne rosacea in Iran has been shown by researchers. Moraveje and Dehghan in a study in 2004 in Gorgan concluded that the presence of Demodex mites increases the possibility of rosacea (1).

Demodicosis can infect both genders in humans, but some studies have reported that women are 2.5 times more likely to be infected. The disease is also more common in people over the age of 40 years $(8,9)$.

In a study conducted in Iran on 100 people, the percentage of infection was $43 \%$ in people under 20 years of age, $66 \%$ in people aged $20-40$ years, and $85 \%$ in people over 40 years of age, indicating a significant relationship between age and prevalence of this infection (10).

In another study by Javidi et al, the relationship between androgenic alopecia and Demodex infestation was investigated. All the participants were between 20-40 years old. Demodex was detected in both patients and controls. Therefore, no significant relationship was found between alopecia and Demodex infestation (5).

Due to the possibility of transmitting this mange through direct contact and the use of shared items such as hat and comb, it is necessary that the possibility of infecting other family members and their treatment should be considered.

\section{Conclusion}

Due to the rarity of the reported case and the lack of exposure of esteemed laboratory colleagues to this case of the disease as well as the lack of relevant educational content in the university curriculum, it is suggested that the epidemiological conditions and socio-economic situation in the region and the possibility of this case be considered. In patients, a training workshop for laboratory colleagues working in the province should be planned by respected officials. Considering the present report, it is suggested that human demodicosis be considered as one of the differential diagnoses of dermatitis.

\section{Conflict of Interests}

The authors declare that they have no conflict of interests.

\section{Ethical Issues}

In this research, ethical considerations have been fully observed.

\section{Acknowledgments}

The cooperation of the staff of the clinic and laboratory of Sina Hospital in Tabriz is hereby thanked and appreciated.

\section{Authors' Contribution}

HM and PM did writing and editing of the manuscript.SBA designed and did data collection. AHAH designed, did data collection and statistical analysis.

\section{Funding}

The authors received no financial support for the research, authorship and publication of this article.

\section{References}

1. Moravej H, Dehghan M. The relation between Demodex mites with incidence of acne rosacea. Journal of Gorgan University of Medical Sciences. 2004;6(13):62-66. [Persian].

2. Saridomichelakis $M N$, Koutinas $A F$, Farmaki $R$, Leontides LS, Kasabalis D. Relative sensitivity of hair pluckings and exudate microscopy for the diagnosis of canine demodicosis. Vet Dermatol. 2007;18(2):138-141. doi:10.1111/j.13653164.2007.00570.x

3. Dolenc-Voljc M, Pohar M, Lunder T. Density of Demodex folliculorum in perioral dermatitis. Acta Derm Venereol. 2005;85(3):211-215. doi:10.1080/00015550510030069

4. Belgheis Zadeh H. Determination of Prevalence of Demodicosis and Dependent Factors. Proceeding of 6th National and the 1th Regional Congress on Parasitology and Parasitic Diseases Razi Research Institute; May 27-29, 2008; Karj - Iran p. 469. [Persian].

5. Javidi Z, Fata A, Maleki M, et al. Evaluation of the relationship between androgenetic alopecia and Demodex infestation. Medical Journal of Mashhad University of Medical Sciences. 2005;48(88):163-170. [Persian].

6. Elston DM. Demodex mites: facts and controversies. Clin Dermatol. 2010;28(5):502-504. doi: 10.1016/j. clindermatol.2010.03.006.

7. Erbağci Z, Ozgöztaşi O. The significance of Demodex folliculorum density in rosacea. Int J Dermatol. 1998;37(6):421425. doi: 10.1046/j.1365-4362.1998.00218.x.

8. Soulsby EJL. Helminths Arthropods and Protozoa of Domesticated Animals London: Baillière Tindall; 1968. p. 4979.

9. Saridomichelakis MN, Koutinas AF, Farmaki R, Leontides LS, Kasabalis D. Relative sensitivity of hair pluckings and exudate microscopy for the diagnosis of canine demodicosis. Vet Dermatol. 2007;18(2):138-141. doi: 10.1111/j.13653164.2007.00570.x

10. Benessahraoui M, Paratte F, Plouvier E, Humbert P, Aubin F. Demodicidosis in a child with xantholeukaemia associated with type 1 neurofibromatosis. Eur J Dermatol. 2003;13(3):311-312. 